\title{
On the Spectrum of the Hierarchical Laplacian
}

\author{
Alexander Bendikov • Paweł Krupski
}

Received: 26 August 2013 / Accepted: 24 April 2014 / Published online: 10 June 2014

(C) The Author(s) 2014. This article is published with open access at Springerlink.com

\begin{abstract}
Let $(X, d)$ be a locally compact separable ultrametric space. We assume that $(X, d)$ is proper, that is, any closed ball $B \subset X$ is a compact set. Given a measure $m$ on $X$ and a function $C(B)$ defined on the set of balls (the choice function) we define the hierarchical Laplacian $L_{C}$ which is closely related to the concept of the hierarchical lattice of F.J. Dyson. $L_{C}$ is a non-negative definite self-adjoint operator in $L^{2}(X, m)$. In this paper we address the following question: How general can be the spectrum $\operatorname{Spec}\left(L_{C}\right) \subseteq \mathbb{R}_{+}$? When $(X, d)$ is compact, $\operatorname{Spec}\left(L_{C}\right)$ is an increasing sequence of eigenvalues of finite multiplicity which contains 0 . Assuming that $(X, d)$ is not compact we show that under some natural conditions concerning the structure of the hierarchical lattice ( $\equiv$ the tree of $d$-balls) any given closed subset $S \subseteq \mathbb{R}_{+}$, which contains 0 as an accumulation point and is unbounded if $X$ is non-discrete, may appear as $\operatorname{Spec}\left(L_{C}\right)$ for some appropriately chosen function $C(B)$. The operator $-L_{C}$ extends to $L^{q}(X, m), 1 \leqq q<\infty$, as Markov generator and its spectrum does not depend on $q$. As an example, we consider the operator $\mathfrak{D}^{\alpha}$ of fractional derivative defined on the field $\mathbb{Q}_{p}$ of $p$-adic numbers.
\end{abstract}

Keywords Hierarchical lattice · Laplacian · Spectrum · Markov semigroup · Ultrametric · P-adic Quantum theory

Mathematics Subject Classifications (2010) 47S10 - 60J25 · 81Q10 · 54E45 05 C05

The research of the first author was supported by the Polish Government Scientific Research Fund, Grant 2012/05/B/ST 1/00613

A. Bendikov · P. Krupski $(\varangle)$

Mathematical Institute, University of Wrocław,

Pl.Grunwaldzki 2/4, 50-384 Wrocław, Poland

e-mail: Pawel.Krupski@math.uni.wroc.pl

A. Bendikov

e-mail: bendikov@math.uni.wroc.pl 


\section{Introduction}

The concept of hierarchical lattice and hierarchical distance was proposed by F.J. Dyson in his famous papers on the phase transition for 1D ferromagnetic model with long range interaction $[5,6]$.

The notion of hierarchical Laplacian $L$, which is closely related to the Dyson's model was studied in several mathematical papers [4], [11-13] and [16].

These papers contain some basic information about $L$ (the spectrum, Markov semigroup, resolvent etc) in the case when the hierarchical lattice satisfies some symmetry conditions (homogeneity, self-similarity etc). Under these symmetry conditions, $\operatorname{Spec}(L)$ is pure point and all eigenvalues have infinite multiplicity. The main goal of the papers mentioned above was to introduce a class of random perturbations of $L$ and then to justify the existence of the spectral bifurcation from the pure point spectrum to the continuous one.

A systematic study of a class of isotropic Markov semigroups defined on an ultrametric space $(X, d)$ has been done in [1] (see also the forthcoming paper [2]). In particular, given an isotropic Markov semigroup $\left(P^{t}\right)$ with Markov generator $-L$, one can show that the operator $L$ is a hierarchical Laplacian on $(X, d)$ associated with an appropriate choice function $C(B)$ and vice versa. Then the general theory developed in [1] and [2] applies: modifying canonically the underlying ultrametric $d$, we call this new ultrametric $d_{*}$, the set $\operatorname{Spec}(L)$ is pure point and can be described as

$$
\operatorname{Spec}(L)=\overline{\left\{\frac{1}{d_{*}(x, y)}: x \neq y\right\}} \cup\{0\} .
$$

In our construction the families of $d$-balls and $d_{*}$-balls coincide, whence these two ultrametrics generate the same topology and the same hierarchical structure, and in particular, the same class of hierarchical Laplacians.

The equation (1.1) leads us to the following question.

\section{(A) How general can be the set $\operatorname{Spec}(L)$ ?}

or equivalently,

(B) How general can be the set $\operatorname{Range}\left(d_{*}\right)$ ?

In the course of the study we assume that $(X, d)$ is a locally compact and separable ultrametric space. Recall that a metric $d$ is called an ultrametric if it satisfies the ultrametric inequality

$$
d(x, y) \leq \max \{d(x, z), d(z, y)\},
$$

that is obviously stronger than the usual triangle inequality. Usually, we also assume that the ultrametric $d$ is proper, that is, each closed $d$-ball is a compact set.

The paper is organized as follows. In Section 2 we recall some basic properties of ultrametric spaces. The main original results there can be summarized in the following statement (see Proposition 2,4, Theorem 2.6, Theorem 2.13).

Theorem 1.1 Let $(X, d)$ be a locally compact, non-compact, separable ultrametric space. Let $M \subset[0, \infty)$ be a countable unbounded set which contains 0 . Assume that if $X$ contains a non-isolated point, then 0 is an accumulation point of $M$. Then the following properties hold:

1. There exists a proper ultrametric $d^{\prime}$ on $X$ which generates the same topology as $d$ and such that Range $\left(d^{\prime}\right)=M$. 
2. Assume that $d$ is proper and that there exists a partition $\Pi$ of $X$ made of $d$-balls which contains infinitely many non-singletons. Then the ultrametric $d^{\prime}$ as above can be chosen such that the collections of $d$-balls and $d^{\prime}$-balls coincide.

Let $\mathcal{B}$ be the set of all non-singleton balls. Let $\mathcal{D}$ be the set of locally constant functions having compact support. In Section 3, given a measure $m$ on $X$ which satisfies some natural conditions and a choice function $C: \mathcal{B} \rightarrow(0, \infty)$, we define (pointwise) the hierarchical Laplacian $\left(L_{C}, \mathcal{D}\right)$ associated with $m$ and $C$,

$$
L_{C} f(x):=-\sum_{B \in \mathcal{B}: x \in B} C(B)\left(P_{B} f-f(x)\right), \quad f \in \mathcal{D},
$$

where

$$
P_{B} f:=\frac{1}{m(B)} \int_{B} f d m .
$$

The operator $\left(L_{C}, \mathcal{D}\right)$ acts on $L^{2}=L^{2}(X, m)$, is symmetric and admits a complete system of eigenfunctions $\left\{f_{B, B^{\prime}}\right\}_{B^{\prime} \in \mathcal{B}}$,

$$
f_{B, B^{\prime}}=\frac{1}{m(B)} \mathbf{1}_{B}-\frac{1}{m\left(B^{\prime}\right)} \mathbf{1}_{B^{\prime}},
$$

where $B \subset B^{\prime}$ are nearest neighboring balls; when $m(X)<\infty$, we also set $f_{X, X^{\prime}}=$ $1 / m(X)$. The eigenvalue $\lambda\left(B^{\prime}\right)$ corresponding to $f_{B, B^{\prime}}$ is

$$
\lambda\left(B^{\prime}\right)=\sum_{T \in \mathcal{B}: B^{\prime} \subseteq T} C(T) ;
$$

when $m(X)<\infty$, we set $\lambda\left(X^{\prime}\right)=0$. In particular, we conclude that $\left(L_{C}, \mathcal{D}\right)$ is an essentially self-adjoint operator in $L^{2}$. By abuse of notation, we shell write $\left(L_{C}\right.$, Dom $\left.L_{C}\right)$ for its unique self-adjoint extension.

Let $B \subset B^{\prime}$ be two nearest neighboring balls. Choosing the function

$$
C(B)=\frac{1}{\operatorname{diam}(B)}-\frac{1}{\operatorname{diam}\left(B^{\prime}\right)}
$$

we obtain

$$
\lambda(B)=\frac{1}{\operatorname{diam}(B)},
$$

for any $B \in \mathcal{B}$. Applying Theorem 1.1, we answer question $(A)$.

Theorem 1.2 Let $(X, d)$ be a locally compact, non-compact, separable ultrametric space. Let $S \subseteq[0, \infty)$ be a closed set which contains 0 as an accumulation point. Assume that if $X$ contains a non-isolated point then $S$ is unbounded. Then the following properties hold:

1. There exist a proper ultrametric $d^{\prime}$ on $X$ which generates the same topology as $d$ and a choice function $C(B)$ defined for $\left(X, d^{\prime}\right)$ such that $\operatorname{Spec}\left(L_{C}\right)=S$.

2. Assume that $d$ is proper and that there exists a partition $\Pi$ of $X$ made of d-balls containing infinitely many non-singletons. Then there exists a choice function $C(B)$ defined for $(X, d)$ such that $\operatorname{Spec}\left(L_{C}\right)=S$.

Actually, we get Theorem 1.2 not only for the particular choice function mentioned above but for more general types of them (see the proof in Section 3 ).

The very simple Example 2.3 shows that the condition "there exists a partition $\Pi$ of $X$ made of $d$-balls containing infinitely many non-singletons" in statement (2) of Theorem 1.1 
and Theorem 1.2 can not be dropped: $X=\mathbb{N}$ and $d(m, n)=\max (m, n)$ when $m \neq n$ and 0 otherwise.

In the concluding Section 4 we consider the operator $\mathfrak{D}^{\alpha}$ of the $p$-adic fractional derivative of order $\alpha>0$. This operator related to the concept of $p$-adic Quantum Mechanics was introduced by V.S. Vladimirov, see [17], [18] and [19]. We prove that $\mathfrak{D}^{\alpha}$ is a hierarchical Laplacian. The main novelty here is that $\mathfrak{D}^{\alpha}$ admits a closed extension in $L^{q}, 1 \leq q<\infty$, call it $\mathfrak{D}_{q}^{\alpha}$. The closed operator $-\mathfrak{D}_{q}^{\alpha}$ coincides with the infinitesimal generator of a translation invariant Markov semigroup acting in $L^{q}$. The set $\operatorname{Spec}\left(\mathfrak{D}_{q}^{\alpha}\right)$ consists of eigenvalues $p^{k \alpha}, k \in \mathbb{Z}$, each of which has infinite multiplicity and contains 0 as an accumulation point. In particular, $\operatorname{Spec}\left(\mathfrak{D}_{q}^{\alpha}\right)=\operatorname{Spec}\left(\mathfrak{D}_{2}^{\alpha}\right)$, for all $1 \leq q<\infty$. We study also random perturbations $\mathfrak{D}^{\alpha}(\omega)$ of the operator $\mathfrak{D}^{\alpha}$ and provide a limit behaviour of its normalized eigenvalues.

\section{Metric Matters}

Recall that a topological space $X$ is totally disconnected if for any two distinct points $x, y \in$ $X$ there exists a closed and open (=clopen) subset $U$ of $X$ such that $x \in U$ and $y \notin U$; if $X$ has a basis consisting of clopen subsets, then $X$ is called zero-dimensional.

Clearly, zero-dimensional spaces are totally disconnected but there are Polish spaces which are totally disconnected and not zero-dimensional (for example, the complete Erdôs space $E=\left\{\left(x_{i}\right) \in l_{2}: x_{i} \in \mathbb{R} \backslash \mathbb{Q}\right\}$, see [7, Section 1.4] ). Nevertheless, for locally compact Hausdorff spaces these two notions coincide, i.e. totally disconnected locally compact Hausdorff spaces are zero-dimensional.

At the beginning let us recall two classical topological characterizations which are crucial in the study of zero-dimensional separable metric spaces (see, e.g., [7, Problem 1.3.F.]).

Proposition 2.1 A topological space $X$ is homeomorphic to the Cantor set $\mathcal{C}=\{0,1\}^{\aleph_{0}}$ if and only if $X$ is metrizable, compact, perfect and totally disconnected.

It is well known that each non-empty locally compact, non-compact, metrizable, separable space $X$ can be compactified by adding an extra point $\omega$ whose neighborhoods are declared to be of the form $\{\omega\} \cup(X \backslash K)$, where $K$ is a compact subset of $X$. One can easily check that $\omega$ has a countable basis of neighborhoods of this form. It follows that the compact space $X \cup\{\omega\}$ has a countable base, so it is metrizable. If, additionally, $X$ has no isolated points (i.e., $X$ is perfect) and is totally disconnected, then $X \cup\{\omega\}$ is homeomorphic to $\mathcal{C}$ by Proposition 2.1. Thus we get the following characterization.

Proposition 2.2 Each metrizable locally compact, non-compact, separable, perfect, totally disconnected space is homeomorphic to $\mathcal{C} \backslash\{p\}$, where $p$ is an arbitrary point of $\mathcal{C}$.

Let us now list some basic properties of ultrametric spaces $(X, d)$ (see [3, p. 227], [14]).

(a) Each ball $B_{r}(a)=\{x \in X: d(x, a)<r\}$ is a closed set, diam $B_{r}(a) \leq r$ and $d(x, y)=\inf \{\operatorname{diam} B: B$ is a ball containing $\{x, y\}\}$.

(b) If $x \in B_{r}(a)$, then $B_{r}(a)=B_{r}(x)$.

(c) If two balls intersect, then one of them is contained in the other.

(d) No infinite ultrametric space is isometric to a subset of the Euclidean space [15, Proposition 3.1]. 
If an ultrametric space $(X, d)$ is separable, then the following facts also hold.

(e) The set Range( $d$ ) of all values of metric $d$ is at most countable.

(f) All distinct balls of a given radius $r>0$ form an at most countable partition of $X$.

If, in addition, $X$ is compact, then

(g) Range (d) has at most one accumulation point which is equal to 0.

It is easy to see that properties (b) and (c) are in fact characteristic for an ultrametric:

- $\quad$ if $d$ is a metric on $X$ satisfying either one of them then d is an ultrametric.

It follows from the above properties that each ultrametric space has a basis of clopen sets, i.e., it is zero-dimensional. Conversely,

- each zero-dimensional separable metrizable space X is metrizable by an ultrametric $d$.

It can be defined in the following way. Let $\left\{B_{1}, B_{2}, \ldots\right\}$ be a basis of clopen subsets of $(X, d)$ and let $f_{n}: X \rightarrow\{0,1\}$ be the characteristic function of $B_{n}, n=1,2, \ldots$ Then

$$
d(x, y)=\max \left\{\frac{\left|f_{n}(x)-f_{n}(y)\right|}{n}: n \in \mathbb{N}\right\}
$$

(see [9]).

The proper ultrametric on $\mathbb{N}$ given in the next example is, in a sense, generic for metrizable, locally compact, non-compact, separable totally disconnected spaces.

Example 2.3 Let $\mathbb{N}$ be equipped with the ultrametric

$$
d_{\max }(m, n)= \begin{cases}\max \{m, n\}, & \text { if } m \neq n \\ 0, & \text { if } m=n\end{cases}
$$

Then any $d_{\max }$-ball is either a singleton or is of the form $\{1,2, \ldots, n\}$.

Proposition 2.4 If $X$ is a metrizable, locally compact, separable, totally disconnected space, then $X$ admits a proper ultrametric that generates the topology of $X$.

Proof There is nothing to prove if $X$ is compact, since any ultrametric metrizing $X$ is automatically proper. Assume that $X$ is not compact. Then there is a partition $\Pi=$ $\left\{P_{1}, P_{2}, \ldots\right\}$ of $X$ made of non-empty, compact-open subsets of $X$. Let $d$ be an ultrametric on $X$ that generates the topology of $X$. We get our proper ultrametric by the following formula:

$$
d_{\Pi}(x, y)= \begin{cases}d(x, y), & \text { if } x, y \in P_{k} \text { for some } k \\ \max (m, n), & \text { if } x \in P_{m}, y \in P_{n} \text { and } m \neq n .\end{cases}
$$

Indeed, observe that $d_{\Pi}\left(P_{1}, P_{n}\right):=\inf \left\{d(x, y): x \in P_{1}, y \in P_{n}\right\} \rightarrow \infty$ if $n \rightarrow \infty$. Hence, each closed $d_{\Pi}$-bounded subset of $X$ is compact.

Lemma 2.5 Let $X$ be a metrizable, locally compact, non-compact, separable, totally disconnected space. There exists an infinite countable partition $\Pi$ of $X$ such that each element of $\Pi$ is a nondegenerate (i.e., non-singleton) compact-open subset of $X$ which contains an accumulation point or, else, it is a two-point set. 
Proof Let us take any infinite, countable partition $\mathcal{R}$ of $X$ consisting of compact-open subsets of $X$ and let

$$
\mathcal{R}^{\prime}=\{R \in \mathcal{R}: R \text { contains no accumulation points }\} .
$$

Clearly, every $R \in \mathcal{R}^{\prime}$ is a finite set. If $\mathcal{R}^{\prime}$ is finite, then choose $R_{0} \in \mathcal{R} \backslash \mathcal{R}^{\prime}$ and define

$$
\Pi=\left\{R_{0} \cup \bigcup \mathcal{R}^{\prime}\right\} \cup\left(\mathcal{R} \backslash\left(\mathcal{R}^{\prime} \cup\left\{R_{0}\right\}\right)\right) .
$$

If $\mathcal{R}^{\prime}$ is infinite, say $\mathcal{R}^{\prime}=\left\{R_{1}, R_{2}, \ldots\right\}$, then there are mutually disjoint two-point sets $P_{1}, P_{2}, \ldots$ such that $\bigcup_{n=1}^{\infty} R_{n}=\bigcup_{n=1}^{\infty} P_{n}$ and we can put

$$
\Pi=\left(\mathcal{R} \backslash \mathcal{R}^{\prime}\right) \cup\left\{P_{1}, P_{2}, \ldots\right\} .
$$

The proof is finished.

Let $(X, d)$ be a compact separable ultrametric space. It follows from property $(\mathrm{g})$ that if $X$ is infinite, we can arrange the values of $d$ in a sequence decreasing to 0, i.e., $\operatorname{Range}(d)=$ $\left\{c_{1}, c_{2}, \ldots\right\}$, where $c_{n} \searrow 0$. Since, for any other sequence $c_{n}^{\prime} \searrow 0$, there is an increasing surjection $\phi:\left\{c_{1}, c_{2}, \ldots\right\} \rightarrow\left\{c_{1}^{\prime}, c_{2}^{\prime}, \ldots\right\}$, we get equivalent proper ultrametric $d^{\prime}=\phi \circ d$ on $X$ with the same family of balls as $d$ and with Range $\left(d^{\prime}\right)=\left\{c_{1}^{\prime}, c_{c}^{\prime}, \ldots\right\}$.

An analogous statement for locally compact, non-compact $(X, d)$ has a more complicated nature.

Theorem 2.6 Let $X$ be a metrizable, locally compact, non-compact, separable, totally disconnected space and $\Pi$ be an infinite countable partition of $X$ as in Lemma 2.5. Let $M \subset[0, \infty)$ be a countable unbounded set containing 0 . Assume that if $X$ contains an accumulation point, then 0 is an accumulation point of $M$. Then, for each proper ultrametric $d_{p}$ which generates the topology of $X$, there exists an equivalent proper ultrametric $d$ on $X$ such that $\operatorname{Range}(d)=M$ and the collections of $d_{p}$-balls and $d$-balls contained in any $P \in \Pi$ coincide. Moreover, $d(x, y) \leq d_{p}(x, y)$ for $x, y \in B$ and each $d_{p}$-ball $B \varsubsetneqq P \in \Pi$.

The idea of the proof of Theorem 2.6 is based on a specific tree-structure of the family of balls in an ultrametric space. So, let us first introduce necessary notions and tools.

Let $\mathcal{T}(X, d)$ be the collection of all balls in a proper ultra-metric space $(X, d)$. Consider $\mathcal{T}(X, d)$ with a partial order $B^{\prime} \preccurlyeq B \Leftrightarrow B \subseteq B^{\prime}$ and a semi-lattice operation $A \wedge B=$ $\inf \{A, B\}$, the infimum taken with respect to $\preccurlyeq$ (which is the smallest, with respect to the inclusion, ball containing balls $A$ and $B)$. We prefer to view $\mathcal{T}(X, d)$ geometrically as a countable, locally finite tree with vertices being elements of $\mathcal{T}(X, d)$ and edges being pairs of $d$-balls $\left(B, B^{\prime}\right)$ such that $B$ is an immediate successor or predecessor of $B^{\prime}$. Vertices with no successor are called end-points of the tree; they represent singleton balls.

Let $2^{Y}$ be the family of compact nonempty subsets of a metric space $(Y, d)$ metrized by the Hausdorff metric

$$
H_{d}(A, B):=\inf \left\{r>0: A \subset N_{d}(r, B) \text { and } B \subset N_{d}(r, A)\right\} \text {, }
$$

where $N_{d}(r, A)=\{x \in Y: d(x, A)<r\}, d(x, A)=\inf \{d(x, a): a \in A\}$.

Definition 2.7 Given $\mathcal{H} \subset 2^{Y}$, a continuous map $w: \mathcal{H} \rightarrow[0, \infty)$ is said to be a Whitney map for $\mathcal{H}$ if

1. $w(A)<w(B)$ whenever $A \varsubsetneqq B$,

2. $w(A)=0$ if and only if $A$ is a singleton. 
Whitney maps for $2^{Y}$ exist for metric separable spaces $Y$ [10, p. 205]. If $(Y, d)$ is a proper ultrametric space, then property (b) implies that the diameter function diam : $\mathcal{T}(Y, d) \rightarrow$ $[0, \infty)$ is a Whitney map.

Denote $F_{1}=\{\{x\}: x \in X\}$. The following proposition will be used in Section 3 .

Proposition 2.8 Let $(X, d)$ be a proper ultrametric space with a Whitney map $w$ : $\mathcal{T}(X, d) \cup F_{1} \rightarrow[0, \infty)$ satisfying the following condition:

$$
\lim _{n \rightarrow \infty} w\left(B_{n}\right)=\infty \quad \text { for each infinite sequence } \quad B_{1} \varsubsetneqq B_{2} \varsubsetneqq \ldots
$$

Then the formula $d_{*}(x, y)=w(\{x\} \wedge\{y\})$ for $x \neq y$ (where $\{x\} \wedge\{y\}$ denotes the smallest ball containing $x$ and $y)$ and $d_{*}(x, y)=0$ for $x=y$ defines a proper ultrametric in $X$ which induces the same topology and the same collection of balls as $d$.

Proof It is easy to verify that $d_{*}$ is an ultrametric. It is equivalent to $d$ by the continuity of $w$ at each singleton. In order to show that $d_{*}$ is proper, let $\overline{B_{r}^{*}}(x)$ be a closed $d_{*}$-ball of radius $r$ centered at $x$. Notice that Eq. (2.1) implies that there is a $d$-ball $B(x)$, centered at $x$, containing $\overline{B_{r}^{*}}(x)$. Since $B(x)$ is compact, we conclude that the ball $\overline{B_{r}^{*}}(x)$ is compact.

Claim 2.9 For any $d$-ball $B(x)$ centered at $x$ there exists $\epsilon>0$ such that $d_{*}$-ball $B_{r(\epsilon)}^{*}(x)$ of radius $r(\epsilon)=w(B(x))+\epsilon$, centered at $x$, is equal to $B(x)$.

Proof of Claim 2.9 Clearly, we can assume that $B(x)$ is nondegenerate. The inclusion $B(x) \subset B_{r(\epsilon)}^{*}(x)$ holds for each $\epsilon>0$, since if $y \in B(x) \backslash\{x\}$, then $\{x\} \wedge\{y\} \subset B(x)$, hence

$$
d_{*}(x, y)=w(\{x\} \wedge\{y\}) \leq w(B(x))<w(B(x))+\epsilon .
$$

Suppose that, for each $\epsilon>0, B(x)$ is a proper subset of $B_{r(\epsilon)}^{*}(x)$. For each $n \in \mathbb{N}$, choose a point $y_{n} \in B_{r\left(\frac{1}{n}\right)}^{*}(x) \backslash B(x)$. Then $B(x) \varsubsetneqq\{x\} \wedge\left\{y_{n}\right\}$ and we get

$$
0<w(B(x))<w\left(\{x\} \wedge\left\{y_{n}\right\}\right)=d_{*}\left(x, y_{n}\right)<w(B(x))+\frac{1}{n} .
$$

The balls $\{x\} \wedge\left\{y_{n}\right\}$ are contained in a branch of $\mathcal{T}(X, d)$, so we can choose a subsequence $n_{k}$ such that balls $\{x\} \wedge\left\{y_{n_{k}}\right\}$ form a decreasing family of sets, in view of Eq. (2.2). So $d_{*}\left(x, y_{n_{k}}\right) \rightarrow w(B(x))$ if $k \rightarrow \infty$ and points $x, y_{n_{k}}, k \in \mathbb{N}$, belong to a compact set $\{x\} \wedge\left\{y_{n_{1}}\right\}$. It means that Range $\left(d_{*}\right)$ has an accumulation point different from 0 on a compact set, contrary to property $(\mathrm{g})$.

It remains to show that each nondegenerate $d_{*}$-ball $B^{*}(x)$ centered at $x$ is a $d$-ball. Let $Z:=\left\{r>0: B^{*}(x)=B_{r}^{*}(x)\right\}$. Obviously $Z \neq \emptyset$. Denote $r^{\prime}=\inf Z$. Notice that $r^{\prime}>0$. We have

$$
B^{*}(x)=\left\{y: d_{*}(x, y) \leq r^{\prime}\right\} .
$$

Indeed, the inclusion $\subset$ in Eq. (2.3) is obvious for any metric. The inclusion $\supset$ is also trivial in the case $r^{\prime} \notin Z$. But the case $r^{\prime} \in Z$ cannot occur because $B^{*}(x)=B_{r^{\prime}}^{*}(x)$ would mean that $r^{\prime}$ is an accumulation point of distances $d_{*}(x, y)$ for points $y \in B^{*}(x)$ in the compact set $\overline{B_{r^{\prime}}^{*}}(x)=\left\{y: d_{*}(x, y) \leq r^{\prime}\right\}$, contrary to property $(\mathrm{g})$. This also shows that

$$
\text { there is } a \in B^{*}(x) \text { satisfying } d_{*}(x, a)=r^{\prime} .
$$

Finally, we can see that

$$
B^{*}(x)=\{x\} \wedge\{a\} \in \mathcal{T}(X, d) .
$$


In fact, we have already shown that the $d$-ball $\{x\} \wedge\{a\}$ is also a $d_{*}$-ball, so either $\{x\} \wedge\{a\} \subset$ $B^{*}(x)$ or $B^{*}(x) \varsubsetneqq\{x\} \wedge\{a\}$.

Suppose the latter inclusion holds. Then, by Eqs. (2.3) and (2.4), there is a point $y \in$ $\{x\} \wedge\{a\}$ with $d_{*}(x, y)>r^{\prime}=d_{*}(x, a)$. Hence, $w(\{x\} \wedge\{y\})>w(\{x\} \wedge\{a\})$, so $\{x\} \wedge\{y\} \supseteq$ $\{x\} \wedge\{a\}$. On the other hand, $y \in\{x\} \wedge\{a\}$ implies that $\{x\} \wedge\{y\} \subset\{x\} \wedge\{a\}$, a contradiction.

Therefore $\{x\} \wedge\{a\} \subset B^{*}(x)$. But the inclusion cannot be strict, since otherwise there is a point $y \in B^{*}(x) \backslash\{x\} \wedge\{a\}$ and we have

$$
w(\{x\} \wedge\{y\})=d_{*}(x, y) \leq r^{\prime}=d_{*}(x, a)=w(\{x\} \wedge\{a\}),
$$

which implies $\{x\} \wedge\{y\} \subset\{x\} \wedge\{a\}$, a contradiction. The proof of Proposition 2.8 is finished.

Proof of Theorem 2.6 We are going to construct a countable, locally finite tree $\mathcal{T} \subset 2^{X}$ without the least element and a Whitney map $w$ for $\mathcal{T} \cup F_{1}$ such that Range $(w)=M$ (recall that $F_{1}$ is the set of all singletons in $X$ ).

Let $\Pi=\left\{P_{1}, P_{2}, \ldots\right\}$. Embed the tree $\mathcal{T}\left(\mathbb{N}, d_{\max }\right)$ from Example 2.3 in $2^{X}$ by a one-toone function $\phi$ such that $\phi(\{n\})=P_{n}$ and $\phi(\{1,2, \ldots, n\})=\bigcup_{i=1}^{n} P_{i}$.

For each $P \in \Pi$, let $\mathcal{T}\left(P, d_{p}\right)$ be the rooted (at $P$ ) tree of closed $d_{p}$-balls of $X$ which are contained in $P$. Observe that if $P$ is a doubleton, then $\mathcal{T}\left(P, d_{p}\right)$ splits into two singletons, otherwise $\mathcal{T}\left(P, d_{p}\right)$ has an infinite branch contained in the set $\widetilde{\mathcal{T}\left(P, d_{p}\right)}$ of non-singleton vertices. Trees $\mathcal{T}\left(P, d_{p}\right)$ extend the tree $\phi\left(\mathcal{T}\left(\mathbb{N}, d_{\text {max }}\right)\right)$ and as a result we obtain a tree $\mathcal{T} \subset 2^{X}$.

To each branch $\mathcal{L}$ of $\mathcal{T}$ there corresponds a point $x_{\mathcal{L}} \in X$ such that $\left\{x_{\mathcal{L}}\right\}=\bigcap \mathcal{L}$ (since $\left(X, d_{p}\right)$ is a complete space) and this correspondence is a bijection between the set of all branches and $X$. Observe that the bijection locally (inside of each $P \in \Pi$ ) preserves $d_{p^{-}}$ balls. The semi-lattice operation $\wedge$ on $\mathcal{T}$ can now be extended over the set of all singletons of $X$ by

$$
\left\{x_{\mathcal{L}}\right\} \wedge\left\{x_{\mathcal{L}^{\prime}}\right\}=\inf \left(\mathcal{L} \cap \mathcal{L}^{\prime}\right) .
$$

We will now construct a Whitney map $w$ for $\mathcal{T} \cup F_{1}$ such that Range $(w)=M$. Enumerate positive numbers in $M$ as $m_{1}, m_{2}, \ldots$ and choose a sequence $\left(k_{n}\right) \in M$ such that

$$
k_{1}>\max \left\{m_{1}, m_{2}\right\}, \quad k_{n+1}>\max \left\{k_{n}, m_{n+1}\right\}
$$

(this can be done since $M$ is unbounded).

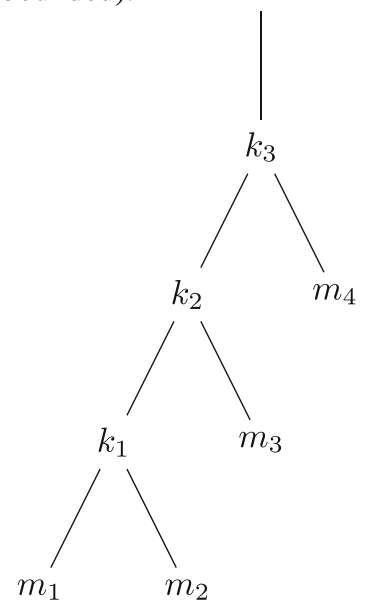


Put $w(B)=0$ if $B$ is a singleton, $w(B)=m_{n}$ if $B=P_{n}$ and $w(B)=k_{n}$ if $B=\bigcup_{i=1}^{n} P_{i}$. It remains to define $w$ on the set $\widetilde{\mathcal{T}\left(P, d_{p}\right)}$ for each $P \in \Pi$ that contains an accumulation point. Then, since 0 must be an accumulation point of $M$ by the hypothesis, there is a strictly decreasing sequence $w(P)>w_{n} \rightarrow 0$ in $M$. Now, take a function

$$
s_{P}: \widetilde{\mathcal{T}\left(P, d_{p}\right)} \backslash\{P\} \rightarrow\left\{w_{1}, w_{2}, \ldots\right\}
$$

which is order preserving on each branch of $\widetilde{\mathcal{T}\left(P, d_{p}\right)}$, i.e., $s_{P}(B)<s_{P}\left(B^{\prime}\right)$ if $B \varsubsetneqq B^{\prime}$, such that $s_{P}(B) \leq \operatorname{diam}_{d_{p}}(B)$ and put

$$
w(B)=s_{P}(B) \quad \text { for } \quad B \in \widetilde{\mathcal{T}\left(P, d_{p}\right)} \backslash\{P\} .
$$

The construction of the Whitney map $w$ is thus complete.

The desired ultrametric $d$ is given by the formula

$$
d(x, y)=w(\{x\} \wedge\{y\}) \text { for } \quad x \neq y \text { and } d(x, y)=0 \text { for } x=y .
$$

Notice that $w(B)=\operatorname{diam} B$ in the metric $d$ and $\mathcal{T}=\mathcal{T}(X, d)$.

Remark 2.10 As we have already remarked in the proof of Theorem 2.6, the trees $\mathcal{T}\left(X, d_{p}\right)$ and $\mathcal{T}(X, d)$ locally coincide, i.e., the collections of $d_{p}$-balls and $d$-balls are the same within each $P \in \Pi$. Whether one can build an ultrametric $d$ on $X$ which satisfies conditions of the theorem and such that collections of all $d_{p}$-balls and $d$-balls coincide is an interesting on its own and useful in applications question, see Section "Hierarchical Laplacian". Example 2.3 shows that, in general, the answer is negative. On the other hand, the answer is positive under the following extra condition:

There is a partition $\Pi$ of $X$ consisting of $d_{p}$-balls and infinitely many of the balls are non-singletons. In terms of the order $\preccurlyeq$ : there is an infinite antichain in $\mathcal{T}\left(X, d_{p}\right)$ (i.e., a subset of $\mathcal{T}\left(X, d_{p}\right)$ whose elements are pairwise incomparable by $\left.\preccurlyeq\right)$ which contains at most finitely many end-points.

Notice that a maximal antichain in $\mathcal{T}\left(X, d_{p}\right)$ is a partition of $X$.

The above condition evidently holds if the ultrametric space $X$ is perfect (or contains at most finitely many isolated points).

The following example is a good illustration of the condition in case of discrete $X$.

Example 2.11 Consider the infinite countable discrete group

$$
X=\bigoplus_{k=1}^{\infty} \mathbb{G}_{k}, \quad \mathbb{G}_{k}=\mathbb{Z}(2)
$$

with the standard ultrametric

$$
d_{p}(x, y)=\min \left\{k: x-y \in G_{k}\right\},
$$

where

$$
G_{0}=\{0\}, \quad G_{k}=\bigoplus_{1 \leq i \leq k} \mathbb{G}_{i} .
$$

All $d_{p}$-balls are either finite subgroups $G_{k}$ or their cosets $G_{k}+g$. The balls form a binary tree $\mathcal{T}(X)$ without the least element and with singletons as its end-points. 
Lemma 2.12 Let $\left(X, d_{p}\right)$ be a separable proper ultrametric space. Suppose there is a partition $\mathcal{S}$ of $X$ consisting of $d_{p}$-balls and infinitely many of the balls are nondegenerate. Then there is a partition $\Pi$ consisting of $d_{p}$-balls with infinitely many nondegenerate members $P$ such that $P$ either contains an accumulation point or all immediate $\preccurlyeq$-successors of $P$ are singletons.

Proof Let $\mathcal{B}=\left\{B_{1}, B_{2}, \ldots\right\} \subset \mathcal{S}$ be the family of all nondegenerate elements of $\mathcal{S}$.

We modify the partition $\mathcal{S}$ as follows. For each $B \in \mathcal{B}$ which contains no accumulation point (i.e., $B$ is finite), choose a point $b \in B$ and let $B(b) \subset B$ be a ball which is an immediate $\preccurlyeq$-predecessor of $\{b\}$. The modified partition $\Pi$ consists of all elements of $\mathcal{S}$ which contain an accumulation point, all balls of the form $B(b)$ and all remaining singletons.

Theorem 2.13 Let $\left(X, d_{p}\right)$ be a separable proper ultrametric space. Suppose there is a partition of $X$ consisting of $d_{p}$-balls and infinitely many of the balls are non-singletons. Then, for every set $M \subset[0, \infty)$ satisfying the hypotheses of Theorem 2.6, there is an equivalent proper ultrametric $d$ on $X$ such that $\operatorname{Range}(d)=M$, the collections of $d_{p}$-balls and $d$-balls coincide and $d \leq d_{p}$ on balls which are proper subsets of those elements of the partition that contain accumulation points.

Proof By Lemma 2.12 there is a partition $\Pi$ such that each nondegenerate element $P \in \Pi$ either contains an accumulation point or all immediate $\preccurlyeq$-successors of $P$ are singletons. Let $\left\{B_{1}, B_{2}, \ldots\right\} \subset \Pi$ be the family of all nondegenerate elements of $\Pi$.

We slightly modify the proof of Theorem 2.6 by considering the original tree of $d_{p}$-balls over partition $\Pi$ instead of tree $\phi\left(\mathcal{T}\left(\mathbb{N}, d_{\text {max }}\right)\right)$.

Let $0=l_{0}<l_{1}<l_{2} \cdots \rightarrow \infty$ be a sequence such that

$$
M_{k}:=M \cap\left(l_{k-1}, l_{k}\right] \neq \emptyset \quad \text { for each } k>0 .
$$

Consider a function $\kappa: M \rightarrow \mathbb{N}$ such that $\kappa(m)$ is the (unique) index satisfying $m \in M_{\kappa(m)}$. Let $M \backslash\{0\}=\left\{m_{1}, m_{2}, \ldots\right\}$.

Let us define a Whitney map $w$ for $\mathcal{T}\left(X, d_{p}\right) \cup F_{1}$. Put $w=0$ for all singletons and let $w\left(B_{i}\right)=m_{i}$. Each $d_{p}$-ball $B$ preceding some $P \in \Pi$ uniquely decomposes into the union of distinct elements of $\Pi$ (one of them is $P$ itself):

$$
B=P_{i_{1}} \cup \cdots \cup P_{i_{n}} \quad \text { for some } i_{1}<\cdots<i_{n} .
$$

For such ball $B$, choose $w(B)$ as a number in $M_{s(B)}$, where

$$
s(B)=\sum_{t=1}^{n} \kappa\left(w\left(P_{i_{t}}\right)\right) .
$$

If a non-singleton ball $B$ succeeds a $P \in \Pi$ in $\mathcal{T}\left(X, d_{p}\right)$, then $P \in \mathcal{B}$ and $B \in \widetilde{\mathcal{T}\left(P, d_{p}\right)}$, so we can define $w(B)$ as in the the proof of Theorem 2.6. The metric

$$
d(x, y)=w(\{x\} \wedge\{y\}) \quad \text { if } \quad x \neq y \quad \text { and } \quad d(x, y)=0 \quad \text { if } \quad x=y
$$

is the required one.

We can also observe that $d(x, y) \leq d_{p}(x, y)$ for $x, y \in B$ and each $d_{p}$-ball $B$ properly contained in $P \in \Pi$. 
Remark 2.14 We can compare Theorem 2.6 with a result in [14, Theorem 2] which says that by a slight change of an ultrametric $d$ in an arbitrary separable ultrametric space $(X, \mathrm{~d})$ one can get an equivalent ultrametric $r \leq d$ that assumes only dyadic rational values. No preservation of balls is discussed in [14].

\section{Hierarchical Laplacian}

The aim of this section is to justify the properties of the hierarchical Laplacian listed in the Introduction. Let $(X, d)$ be a locally compact, separable, proper ultrametric space. Let $m$ be a Radon measure on $X$ such that $m(B)>0$ for each ball $B$ of positive diameter and such that $m(\{x\})=0$ if and only if $x$ is a non-isolated point. Let $\mathcal{D}$ be the set of locally constant functions having compact support.

Definition 3.1 A choice function $C(B)$ is a function defined on the set $\mathcal{B}$ of all non-singleton balls $B$, taking values in $(0, \infty)$ and such that

1. $\lambda(B):=\sum_{T \in \mathcal{B}: B \subseteq T} C(T)<\infty$.

2. $\lim _{B \downarrow\{x\}} \lambda(B)=\infty$ if $x$ is not an isolated point.

Given a choice function $C(B)$ and a measure $m$ as above we consider the hierarchical Laplacian $\left(L_{C}, \mathcal{D}\right)$ defined pointwise by the Eq. (1.3), that is,

$$
L_{C} f(x):=-\sum_{B \in \mathcal{B}: x \in B} C(B)\left(P_{B} f-f(x)\right) .
$$

Lemma $3.2\left(L_{C}, \mathcal{D}\right)$ acts in all spaces $L^{p}, 1 \leq p \leq \infty$.

Proof Since the intersection $L^{1} \cap L^{\infty}$ is a subset of each $L^{p}, p>1$, it is enough to prove the claim if $p$ equals 1 and $\infty$. For any ball $T$ of positive measure we set $f_{T}=\mathbf{1}_{T} / \mathrm{m}(T)$ and compute $L_{C}\left(f_{T}\right)(x)$,

$$
\begin{aligned}
L_{C}\left(f_{T}\right)(x) & =-\sum_{x \in B} C(B)\left(P_{B}\left(f_{T}\right)-f_{T}(x)\right) \\
& =-\left(\sum_{x \in B, T \subseteq B}+\sum_{x \in B, B \cap T=\varnothing}+\sum_{x \in B, B \subset T}\right) C(B)\left(P_{B}\left(f_{T}\right)-f_{T}(x)\right) .
\end{aligned}
$$

Next observe that for any ball $B$ centered at $x$,

$$
P_{B}\left(f_{T}\right)=\left\{\begin{array}{clc}
f_{T}(x) & \text { if } & B \subseteq T \\
f_{B}(x) & \text { if } & T \subseteq B \\
0 & \text { if } & B \cap T=\varnothing
\end{array} .\right.
$$

It follows that

$$
\begin{aligned}
L_{C}\left(f_{T}\right)(x) & =-\sum_{x \in B, T \subseteq B} C(B)\left(f_{B}(x)-f_{T}(x)\right) \\
& =\left(\sum_{x \in B, T \subseteq B} C(B)\right) f_{T}(x)-\sum_{x \in B, T \subseteq B} C(B) f_{B}(x) .
\end{aligned}
$$


Clearly we have

$$
\left(\sum_{x \in B, T \subseteq B} C(B)\right) f_{T}(x)=\lambda(T) f_{T}(x),
$$

whence

$$
L_{C}\left(f_{T}\right)(x)=\lambda(T) f_{T}(x)-\sum_{x \in B, T \subseteq B} C(B) f_{B}(x) .
$$

Evidently, $u_{1}=\lambda(T) f_{T}$ is in $L^{1} \cap L^{\infty}$. For the second term in Eq. (3.1), call it $u_{2}$, we have

$$
\begin{aligned}
u_{2}(x) & =\sum_{B:\{x\} \wedge T \subseteq B} C(B) f_{B}(x)=\sum_{B:\{x\} \wedge T \subseteq B} \frac{C(B)}{m(B)} \\
& \leq \frac{1}{m(T)} \sum_{B:\{x\} \wedge T \subseteq B} C(B) \leq \frac{\lambda(T)}{m(T)} .
\end{aligned}
$$

Let $T:=T_{0} \subset T_{1} \subset T_{2} \subset \ldots \subset X$ be an increasing sequence of balls such that each $T_{l+1}$ is the immediate predecessor of $T_{l}$. We set $T_{-1}=\varnothing$ and write

$$
\begin{aligned}
\int_{X} u_{2} d m & =\sum_{i=0}^{\infty} \int_{T_{i} \backslash T_{i-1}} u_{2} d m \\
& =m(T) \sum_{B: T \subseteq B} \frac{C(B)}{m(B)}+\left(m\left(T_{1}\right)-m(T)\right) \sum_{B: T_{1} \subseteq B} \frac{C(B)}{m(B)}+\ldots .
\end{aligned}
$$

Applying the Abel transformation we obtain

$$
\begin{aligned}
\int u_{2} d m= & \lim _{l \rightarrow \infty}\left[\sum_{i=0}^{l-1} m\left(T_{i}\right)\left(\sum_{B: T_{i} \subseteq B} \frac{C(B)}{m(B)}-\sum_{B: T_{i+1} \subseteq B} \frac{C(B)}{m(B)}\right)\right. \\
& \left.+m\left(T_{l}\right) \sum_{B: T_{l} \subseteq B} \frac{C(B)}{m(B)}\right] \\
= & \lim _{l \rightarrow \infty}\left(\sum_{i=0}^{l-1} C\left(T_{i}\right)+m\left(T_{l}\right) \sum_{B: T_{l} \subseteq B} \frac{C(B)}{m(B)}\right)=\lambda(T),
\end{aligned}
$$

whence, in particular, $u_{2}$ is in $L^{1}$. All the above shows that $L_{C}\left(f_{T}\right)$ belongs to both $L^{1}$ and $L^{\infty}$. This finishes the proof since any locally constant function with compact support is a finite linear combination of the functions $f_{T}$.

Let $\left\{f_{B, B^{\prime}}\right\}_{B^{\prime} \in \mathcal{B}}$ be the family of functions defined by Eq. (1.4), i.e.,

$$
f_{B, B^{\prime}}=\frac{1}{m(B)} \mathbf{1}_{B}-\frac{1}{m\left(B^{\prime}\right)} \mathbf{1}_{B^{\prime}}
$$

It is easy to see that all functions $f_{B, B^{\prime}} \in \mathcal{D}$ and that for any two distinct balls $B^{\prime}$ and $C^{\prime}$ the functions $f_{B, B^{\prime}}$ and $f_{C, C^{\prime}}$ are orthogonal in $L^{2}=L^{2}(X, m)$.

Proposition 3.3 In the above notation the following properties hold.

1. $\left\{f_{B, B^{\prime}}\right\}_{B^{\prime} \in \mathcal{B}}$ is a complete system in $L^{2}$. 
2. $L_{C}\left(f_{B, B^{\prime}}\right)(x)=\lambda\left(B^{\prime}\right) f_{B, B^{\prime}}(x)$, for any $x \in X$ and $B^{\prime} \in \mathcal{B}$.

In particular, $\left(L_{C}, \mathcal{D}\right)$ is a non-negative definite essentially self-adjoint operator in $L^{2}$. By abuse of notation, we shell write $\left(L_{C}\right.$, Dom $\left.L_{C}\right)$ for its unique self-adjoint extension.

Proof For the first claim, consider $f_{B}=\mathbf{1}_{B} / m(B)$ for any ball $B$ of positive measure and observe that the series

$$
f_{B}=\sum_{T: B \subseteq T} f_{T, T^{\prime}}
$$

converges pointwise, and since

$$
\sum_{T: B \subseteq T}\left\|f_{T, T^{\prime}}\right\|^{2}=\sum_{T: B \subseteq T}\left(\frac{1}{m(T)}-\frac{1}{m\left(T^{\prime}\right)}\right)=\left\|f_{B}\right\|^{2},
$$

the series (3.2) converges in $L^{2}$ as well. This evidently proves the claim.

For the second claim, fix a couple of closest neighbors $T \subset T^{\prime}$ and write the equation (3.1) for both $T$ and $T^{\prime}$. Subtracting the $T^{\prime}$-equation from the $T$-equation we obtain

$$
\begin{aligned}
L_{C}\left(f_{T, T^{\prime}}\right)(x) & =L_{C}\left(f_{T}\right)(x)-L_{C}\left(f_{T^{\prime}}\right)(x) \\
& =\lambda\left(T^{\prime}\right) f_{T}(x)-\lambda\left(T^{\prime}\right) f_{T^{\prime}}(x)=\lambda\left(T^{\prime}\right) f_{T, T^{\prime}}(x) .
\end{aligned}
$$

as desired.

The operator $\left(L_{C}, \mathcal{D}\right)$ acts in $L^{2}$ by Lemma 3.2, its symmetry follows by inspection. Since $\left(L_{C}, \mathcal{D}\right)$ has a complete system of eigenfunctions, it is essentially self-adjoint, i.e. it admits a unique self-adjoint extension. The proof is finished.

The modified ultrametric $d_{*}$ associated with the operator $\left(L_{C}, \mathcal{D}\right)$ is defined by

$$
d_{*}(x, y)=\left\{\begin{array}{cl}
1 / \lambda(\{x\} \wedge\{y\}) & \text { if } x \neq y \\
0 & \text { if } x=y
\end{array} .\right.
$$

Observe that the function $w: \mathcal{T}(X, d) \cup F_{1} \rightarrow[0, \infty), w(B):=1 / \lambda(B)$ and $w=0$ at each singleton, is a Whitney map. By Proposition $2.8, d_{*}$ is a proper ultrametric which induces the same topology and the same collection of balls as $d$ and, as one easily verifies,

$$
\lambda(B)=\frac{1}{\operatorname{diam}_{*}(B)} .
$$

Proof of Theorem 1.2 Assume an ultrametric space $(X, d)$ and a set $S \subseteq[0, \infty)$ satisfy the hypotheses of Theorem 1.2. Let $\mathrm{M}$ be a countable dense subset of $S^{-1} \cup\{0\}$ containing 0 , where $S^{-1}=\left\{s^{-1}: s \in S, s>0\right\}$. Let $d^{\prime}$ be an equivalent metric with $\operatorname{Range}\left(d^{\prime}\right)=M$, as guaranteed by Theorem 1.1 and let $L_{C}$ be the hierarchical Laplacian on the ultrametric space $\left(X, d^{\prime}\right)$ corresponding to a choice function $C(B)$ such that Range $\left(d^{\prime}\right)=\operatorname{Range}\left(d_{*}^{\prime}\right)$. We can choose, for instance, $C(B)=1 / \operatorname{diam}^{\prime}(B)-1 / \operatorname{diam}^{\prime}\left(B^{\prime}\right)$ where ball $B^{\prime}$ is the immediate predecessor of $B$. Then we have

$$
S=\overline{M^{-1}}=\overline{\left\{1 / d^{\prime}(x, y) ; x \neq y\right\}}=\overline{\left\{1 / d_{*}^{\prime}(x, y) ; x \neq y\right\}}=\operatorname{Spec}\left(L_{C}\right)
$$

which completes the proof of Theorem 1.2.

Let $P_{t}=\exp \left(-t L_{C}\right), t \geq 0$, be a symmetric contraction semigroup generated by the self-adjoint operator $\left(L_{C}\right.$, Dom $\left._{L_{C}}\right)$. 
Proposition 3.4 The semigroup $\left\{P_{t}\right\}$ has the following representation

$$
P_{t} f(x)=\int_{0}^{\infty} P_{B_{r}(x)} f d \sigma^{t}(r), f \in L^{2},
$$

where $\sigma^{t}(r)=\exp (-t / r)$ and $B_{r}(x)$ is the $d_{*}$-ball of radius $r$ centered at $x$.

In particular, $\left\{P_{t}\right\}$ is an isotropic Markov semigroup on the ultrametric measure space $\left(X, d_{*}, m\right)$ as defined and studied in [2].

Proof We choose $f=f_{B}$ and compute $P_{t} f(x)$. Using the identity (3.2) we obtain

$$
\begin{aligned}
P_{t} f(x) & =\sum_{T: B \subseteq T} P_{t} f_{T, T^{\prime}}(x)=\sum_{T: B \subseteq T} e^{-t \lambda\left(T^{\prime}\right)} f_{T, T^{\prime}}(x) \\
& =\sum_{T: B \subseteq T, x \in T^{\prime}} e^{-t \lambda\left(T^{\prime}\right)}\left(f_{T}(x)-f_{T^{\prime}}(x)\right) \\
& =\sum_{T: B \wedge\{x\} \subseteq T} e^{-t \lambda\left(T^{\prime}\right)}\left(f_{T}(x)-f_{T^{\prime}}(x)\right)-e^{-t \lambda(B \wedge\{x\})} f_{B \wedge\{x\}}(x) .
\end{aligned}
$$

Next observe that for any ball $T$ centered at $x$,

$$
P_{T}\left(f_{B}\right)=\left\{\begin{array}{clc}
f_{B}(x) & \text { if } \quad T \subseteq B \\
f_{T}(x) & \text { if } \quad B \subseteq T \\
0 & \text { if } B \cap T=\varnothing
\end{array} .\right.
$$

With this observation in mind we write the equality from above as

$$
P_{t} f(x)=\sum_{T: x \in T} e^{-t \lambda\left(T^{\prime}\right)}\left(P_{T}\left(f_{B}\right)(x)-P_{T^{\prime}}\left(f_{B}\right)(x)\right) .
$$

Applying the Abel transformation and the definition (3.3) of the modified ultrametric $d_{*}$ we get the desired equality with $f=f_{B}$. The set spanned by the functions $f_{B}$ is dense in $L^{2}$, the result follows.

$L^{p}$-Spectrum of the hierarchical Laplacian Consider the semigroup $P_{t}=\exp \left(-t L_{C}\right)$. As $\left\{P_{t}\right\}$ is symmetric and Markovian, it admits an extension to $L^{q}, 1 \leq q<\infty$, as a continuous contraction semigroup, call it $\left\{P_{t}^{q}\right\}$,

$$
P_{t}^{q} f(x)=\int_{0}^{\infty} P_{B_{r}(x)} f d \sigma^{t}(r), f \in L^{q} .
$$

Let $\left(-\mathcal{L}, \operatorname{Dom}_{\mathcal{L}}\right)$ be the infinitesimal generator of the semigroup $\left\{P_{t}^{q}\right\}$. Since the operator $\left(L_{C}, \mathcal{D}\right)$ acts in $L^{q}$ and $\left\{P_{t}^{q}\right\}$ extends $\left\{P_{t}\right\}$, the operator $\mathcal{L}$ defines a closed extension of $L_{C}$, call it $L_{C}^{q}$. Applying Theorem 7.8 in [2] we obtain

Proposition 3.5 For any $1 \leq q<\infty$, the operator $\left(L_{C}, \mathcal{D}\right)$ acting in $L^{q}$ admits a closed extension $L_{C}^{q}$. The closed operator $-L_{C}^{q}$ coincides with the infinitesimal generator of a Markov semigroup acting in $L^{q}$. Moreover, for all $1 \leq q<\infty$,

$$
\operatorname{Spec}\left(L_{C}^{q}\right)=\operatorname{Spec}\left(L_{C}^{2}\right)=\overline{\{\lambda(B): B \in \mathcal{B}\}} \cup\{0\} .
$$




\section{4 p-Adic Fractional Derivative}

Consider the field $\mathbb{Q}_{p}$ of $p$-adic numbers endowed with the $p$-adic norm $\|x\|_{p}$ and the $p$ adic ultrametric $d(x, y)=\|x-y\|_{p}$. Let $m$ be the normalized Haar measure on $\mathbb{Q}_{p}$, that is, $m\left(\mathbb{Z}_{p}\right)=1$, where $\mathbb{Z}_{p}$ is the set of $p$-adic integers.

In the ultrametric space $\left(\mathbb{Q}_{p}, d\right)$ all $d$-balls are either compact subgroups $p^{k} \mathbb{Z}_{p}$ or their cosets $p^{k} \mathbb{Z}_{p}+a$; $\operatorname{diam}\left(p^{k} \mathbb{Z}_{p}+a\right)=p^{-k}$ and $m\left(p^{k} \mathbb{Z}_{p}+a\right)=p^{-k}$. The balls form a regular tree $\mathcal{T}_{p}(X)$ of forward degree $p$ without the least element and without end-points.

The notion of $p$-adic fractional derivative related to the concept of $p$-adic Quantum Mechanics was introduced in several mathematical papers, see Vladimirov [17], Vladimirov and Volovich [18], Vladimirov, Volovich and Zelenov [19]. In particular, in [17] a oneparametric family $\left\{\left(\mathfrak{D}^{\alpha}, \mathcal{D}\right)\right\}_{\alpha>0}$ of operators (called operators of fractional derivative of order $\alpha$ ) have been introduced. Recall that $\mathcal{D}$ is the set of all locally constant functions having compact support.

The operators $\mathfrak{D}^{\alpha}$ were defined via Fourier transform available on the locally compact Abelian group $\mathbb{Q}_{p}$,

$$
\widetilde{\mathfrak{D}^{\alpha}} u(\xi)=\|\xi\|_{p}^{\alpha} \widetilde{u}(\xi)
$$

Moreover, it was shown that each operator $\mathfrak{D}^{\alpha}$ can be written as a Riemann-Liouville type singular integral operator

$$
\mathfrak{D}^{\alpha} u(x)=\frac{p^{\alpha}-1}{1-p^{-\alpha-1}} \int_{\mathbb{Q}_{p}} \frac{u(x)-u(y)}{\|x-y\|_{p}^{1+\alpha}} d m(y) .
$$

The aim of this section is to illustrate the results of Section 3 showing that the operator $\left(\mathfrak{D}^{\alpha}, \mathcal{D}\right)$ is in fact a hierarchical Laplacian. More precisely, we claim that $\left(\mathfrak{D}^{\alpha}, \mathcal{D}\right)$ is a hierarchical Laplacian corresponding to the choice function

$$
C(B)=\left(1-p^{-\alpha}\right) \operatorname{diam}(B)^{-\alpha},
$$

or equivalently, the eigenvalues $\lambda(B)$ are of the form

$$
\lambda(B)=\operatorname{diam}(B)^{-\alpha} .
$$

In particular, the set $\operatorname{Spec}\left(\mathfrak{D}^{\alpha}\right)$ consists of eigenvalues $p^{k \alpha}, k \in \mathbb{Z}$, each of which has infinite multiplicity and contains 0 as an accumulation point.

To prove the claim observe that the Fourier transform $\mathcal{F}: f \mapsto \tilde{f}$ on the locally compact Abelian group $\mathbb{Q}_{p}$ is a linear isomorphism from $\mathcal{D}$ onto itself. This basic fact and (4.1) imply that $\left(\mathfrak{D}^{\alpha}, \mathcal{D}\right)$ is an essentially self-adjoint and non-negative definite operator in $L^{2}=$ $L^{2}\left(\mathbb{Q}_{p}, m\right)$. Next we claim that the spectrum of the symmetric operator $\left(\mathfrak{D}^{\alpha}, \mathcal{D}\right)$ coincides with the range of the function $\xi \mapsto\|\xi\|_{p}^{\alpha}$, that is,

$$
\operatorname{Spec}\left(\mathfrak{D}^{\alpha}\right)=\left\{p^{k \alpha}: k \in \mathbb{Z}\right\} \cup\{0\} ;
$$

the eigenspace $\mathcal{H}(\lambda)$ corresponding to the eigenvalue $\lambda=p^{k \alpha}$, is spanned by the function

$$
f_{k}=\frac{1}{m\left(p^{k} \mathbb{Z}_{p}\right)} \mathbf{1}_{p^{k} \mathbb{Z}_{p}}-\frac{1}{m\left(p^{k-1} \mathbb{Z}_{p}\right)} \mathbf{1}_{p^{k-1}} \mathbb{Z}_{p}
$$

and all its shifts $f_{k}(\cdot+a)$ with $a \in \mathbb{Q}_{p} / p^{k} \mathbb{Z}_{p}$.

Indeed, the ball $B_{s}(0), p^{l} \leq s<p^{l+1}$, is the compact subgroup $p^{-l} \mathbb{Z}_{p}$ of $\mathbb{Q}_{p}$, whence the measure $\omega_{s}=\mathbf{1}_{B_{s}(0)} \mathrm{m} / \mathrm{m}\left(B_{s}(0)\right)$ coincides with the normed Haar measure of that compact subgroup. Since for any locally compact Abelian group, the Fourier transform of the normed Haar measure of any compact subgroup is the indicator of its annihilator group 
and, in our particular case, the annihilator of the group $p^{-l} \mathbb{Z}_{p}$ is the group $p^{l} \mathbb{Z}_{p}$, (see [8]), we obtain

$$
\widetilde{\omega}_{s}(\xi)=\mathbf{1}_{p^{l} \mathbb{Z}_{p}}(\xi)=\mathbf{1}_{\left\{\|\xi\|_{p} \leq p^{-l}\right\}}, \quad \text { where } p^{l} \leq s<p^{l+1} .
$$

Computing now the Fourier transform of the function $f_{k}$,

$$
\tilde{f}_{k}(\xi)=\mathbf{1}_{\left\{\|\xi\|_{p} \leq p^{k}\right\}}-\mathbf{1}_{\left\{\|\xi\|_{p} \leq p^{k-1}\right\}}=\mathbf{1}_{\left\{\|\xi\|_{p}=p^{k}\right\}},
$$

we get

$$
\widetilde{\mathfrak{D}^{\alpha} f_{k}}(\xi)=\|\xi\|_{p}^{\alpha} \widetilde{f}_{k}(\xi)=p^{k \alpha} \widetilde{f}_{k}(\xi)
$$

as desired.

Finally, we apply Proposition 3.3 to conclude that the essentially self-adjoint operator $\left(\mathfrak{D}^{\alpha}, \mathcal{D}\right)$ coincides with the hierarchical Laplacian $\left(L_{C}, \mathcal{D}\right)$ with $C(B)$ given by the equation (4.2).

At last, applying Proposition 3.5 we obtain the following result.

Proposition 4.1 For any $1 \leq q<\infty$, the operator $\mathfrak{D}^{\alpha}$ admits a closed extension $\mathfrak{D}_{q}^{\alpha}$ in $L^{q}$. The closed operator $-\mathfrak{D}_{q}^{\alpha}$ coincides with the infinitesimal generator of a translation invariant Markov semigroup acting in $L^{q}$. Moreover, for all $1 \leq q<\infty$,

$$
\operatorname{Spec}\left(\mathfrak{D}_{q}^{\alpha}\right)=\operatorname{Spec}\left(\mathfrak{D}_{2}^{\alpha}\right) .
$$

In the general setting of Propositions 3.3 and 3.5, some eigenvalues may well have finite multiplicity and some not. Indeed, attached to each ball $B$ of $d_{*}$-diameter $1 / \lambda$ there are the eigenvalue $\lambda$ and the corresponding finite dimensional eigenspace $\mathcal{H}_{B}$. This eigenspace is spanned by the finitely many functions

$$
f_{C, B}=\frac{1}{m(C)} \mathbf{1}_{C}-\frac{1}{m(B)} \mathbf{1}_{B},
$$

where $C$ runs through all balls whose predecessor is $C^{\prime}=B$. Recall that $\operatorname{dim} \mathcal{H}_{B}=l(B)-$ 1 , where $l(B)=\sharp\left\{C \in \mathcal{B}: C^{\prime}=B\right\}$.

It follows that in general, if there exists only a finite number of distinct balls of $d_{*}$ diameter $1 / \lambda$ then the eigenvalue $\lambda$ has finite multiplicity.

This is clearly not the case for the ultrametric measure space $\left(\mathbb{Q}_{p}, d, m\right)$ and the operator $\mathfrak{D}^{\alpha}$. Indeed, every $d_{*}$-ball has its diameter in the set $\Lambda_{\alpha}=\left\{p^{k \alpha}: k \in \mathbb{Z}\right\}$, and each ball $B_{1 / \lambda}(0)$ centered at the neutral element 0 and of diameter $1 / \lambda$ has infinitely many disjoint translates $\left\{a_{i}+B_{1 / \lambda}(0)=B_{1 / \lambda}\left(a_{i}\right), i=1,2, \ldots\right.$, which cover $\mathbb{Q}_{p}$ and are balls of the same diameter. Thus, all eigenvalues have infinite multiplicity.

Remark 4.2 Let $\mathcal{H}(\lambda)$ be the eigenspace corresponding to the eigenvalue $\lambda \in \Lambda_{\alpha}$. Then

$$
L^{2}=\bigoplus_{\lambda \in \Lambda_{\alpha}} \mathcal{H}(\lambda) \quad \text { and } \quad \mathcal{H}(\lambda)=\bigoplus_{i=1}^{\infty} \mathcal{H}_{a_{i}+B_{1 / \lambda}(0)}
$$

We choose for each closed ball $B \subset \mathbb{Q}_{p}$ an orthonormal basis $\left\{e_{i}^{B}: 1 \leq i \leq p-1\right\}$ in $\mathcal{H}_{B}$. In view of Eq. (4.3), the set of eigenfunctions $\left\{e_{i}^{B}: B \in \mathcal{B}, 1 \leq i \leq p-1\right\}$ is an orthonormal basis in $L^{2}$. (This reasoning applies to arbitrary ultrametric spaces.) Whether this set is a Schauder basis in $L^{q}, 1 \leq q<\infty$, is an open question.

Random perturbations Let $\mathfrak{D}$ be the operator of fractional derivative of order $\alpha=1$ acting on the ultrametric measure space $\left(\mathbb{Q}_{p}, d, m\right)$. For simplicity we assume that $p=2$. Let 
$\{\varepsilon(B): B \in \mathcal{B}\}$ be i.i.d. Bernoulli random variables defined on a probability space $(\Omega, P)$. We define a perturbation $\mathfrak{D}(\omega), \omega \in \Omega$, of the operator $\mathfrak{D}$ as follows

$$
\mathfrak{D}(\omega) f(x)=-\sum_{B \in \mathcal{B}: x \in B} C(B, \omega)\left(P_{B} f-f(x)\right), f \in \mathcal{D},
$$

where the perturbated choice function $C(B, \omega)=C(B)(1+\delta \varepsilon(B))$ with $C(B)$ given at Eq. (4.2), $\alpha=1$. Evidently the operator $\mathfrak{D}(\omega)$ is a hierarchical Laplacian, for each $\omega \in \Omega$.

Proposition 4.3 In the notation introduced above,

$$
\operatorname{Spec}(\mathfrak{D}(\omega))=\{0\} \cup\left\{\cup_{l \in \mathbb{Z}}\left[2^{-l}, 2^{-l}(1+\delta)\right]\right\}, \quad P-\text { a.s. }
$$

In particular, when $0<\delta<1$, the set $\operatorname{Spec}(\mathfrak{D}(\omega))$ consists of disjoint intervals and $\{0\}$ whereas, when $\delta \geq 1, \operatorname{Spec}(\mathfrak{D}(\omega))=\mathbb{R}_{+}, P$ - a.s. .

Proof Let $\mathcal{B}_{l} \subset \mathcal{B}$ be the family of all balls of diameter $2^{l}$. For $B \in \mathcal{B}_{l}$ and $\omega \in \Omega$, let us compute the eigenvalue $\lambda(B, \omega)$ of the operator $\mathfrak{D}(\omega)$,

$$
\begin{aligned}
\lambda(B, \omega) & =\sum_{T \in \mathcal{B}: B \subseteq T} C(T, \omega)=\lambda(B)+\delta \sum_{T \in \mathcal{B}: B \subseteq T} \varepsilon(T, \omega) C(T) \\
& =\lambda(B)\left(1+\delta \frac{C(B)}{\lambda(B)} \sum_{T \in \mathcal{B}: B \subseteq T} \frac{C(T)}{C(B)} \varepsilon(T, \omega)\right) \\
& =2^{-l}(1+\delta U(B, \omega)),
\end{aligned}
$$

where

$$
U(B, \omega)=\sum_{k \geq 1} 2^{-k} \varepsilon\left(B_{k}, \omega\right) \quad \text { and } \quad B=B_{1} \varsubsetneqq B_{2} \varsubsetneqq \cdots .
$$

Notice that $U(B), B \in \mathcal{B}_{l}$, are (dependent) identically distributed random variables having values in the interval $[0,1]$.

We claim that

$$
P\left\{\omega: U(B, \omega) \in I \quad \text { for all } \quad B \in \mathcal{B}_{l}\right\}=0,
$$

for any dyadic interval $I \varsubsetneqq[0,1]$.

Indeed, for any given $B \in \mathcal{B}_{l}$,

$$
\{\omega: U(B, \omega) \in I\}=\left\{\omega: \varepsilon\left(B_{k}, \omega\right)=\varepsilon_{k} \text { for all } k \leq \kappa\right\},
$$

for some $\varepsilon_{k}=\varepsilon_{k}(I) \in\{0,1\}$ and $\kappa=\kappa(I)$. Let $\mathcal{B}_{l}^{\prime} \subset \mathcal{B}_{l}$ be an infinite collection of balls such that, for each two balls $A$ and $B$ in $\mathcal{B}_{l}^{\prime}$, the ball $A \wedge B$ belongs to $\mathcal{B}_{l+\kappa}$. Since $\{\varepsilon(B): B \in \mathcal{B}\}$ are i.i.d., we obtain

$$
\begin{aligned}
P\{\omega & \left.: U(B, \omega) \in I \quad \text { for all } B \in \mathcal{B}_{l}\right\} \\
& \leq P\left\{\omega: U(B, \omega) \in I \quad \text { for all } B \in \mathcal{B}_{l}^{\prime}\right\} \\
& =P\left\{\omega: \varepsilon\left(B_{k}, \omega\right)=\varepsilon_{k} \quad \text { for all } \quad k \leq \kappa \text { and } B \in \mathcal{B}_{l}^{\prime}\right\} \\
& =\prod_{B \in \mathcal{B}_{l}^{\prime}} P\left\{\omega: \varepsilon\left(B_{k}, \omega\right)=\varepsilon_{k} \quad \text { for all } \quad k \leq \kappa\right\}=0,
\end{aligned}
$$

as claimed.

At last, by Eq. (4.5), for any given dyadic interval $J \subset[0,1]$, we have

$$
P\left\{\omega: U(B, \omega) \in J \quad \text { for some } \quad B \in \mathcal{B}_{l}\right\}=1 .
$$


The equations (4.6) and (4.4) yield

$$
\overline{\left\{\lambda(B, \omega): B \in \mathcal{B}_{l}\right\}}=\left[2^{-l}, 2^{-l}(1+\delta)\right] \quad P-\text { a.s. },
$$

as desired. The proof is finished.

Let, as in the proof of Proposition 4.3 (Fig. 1),

$$
U(B, \omega)=\sum_{k \geq 1} 2^{-k} \varepsilon\left(B_{k}, \omega\right) \quad \text { and } \quad B=B_{1} \varsubsetneqq B_{2} \varsubsetneqq \cdots .
$$

Choose a reference point $o$ and let $B_{L} \in \mathcal{B}_{L}$ be the ball centred at $o$. Fix a level $\mathcal{B}_{l}$ and define the arithmetic mean $\bar{U}\left(B_{L}, \omega\right), L>l$, as

$$
\bar{U}\left(B_{L}, \omega\right)=\frac{1}{2^{L-l}} \sum_{B: B \subset B_{L}, B \in \mathcal{B}_{l}} U(B, \omega) .
$$

Let $\mathrm{E}[\varepsilon(B)]=p$ and $\operatorname{Var}[\varepsilon(B)]=p q, p+q=1$. Using the tree-structure of the ultrametric measure space $\left(\mathbb{Q}_{2}, d, m\right)$ and the fact that $\{\varepsilon(B): B \in \mathcal{B}\}$ are i.i.d. Bernoulli random variables we compute

1. $\mathrm{E}\left[\bar{U}\left(B_{L}\right)\right]=p$,

2. $\operatorname{Var}\left[\bar{U}\left(B_{L}\right)\right] \sim p q / 2^{L-l}$, as $L \rightarrow \infty$.

In particular, $\sum_{L \geq l} \operatorname{Var}\left[\bar{U}\left(B_{L}\right)\right]<\infty$. It follows that, as $L \rightarrow \infty$,

$$
\bar{U}\left(B_{L}, \omega\right) \longrightarrow p \quad P-a . s .
$$

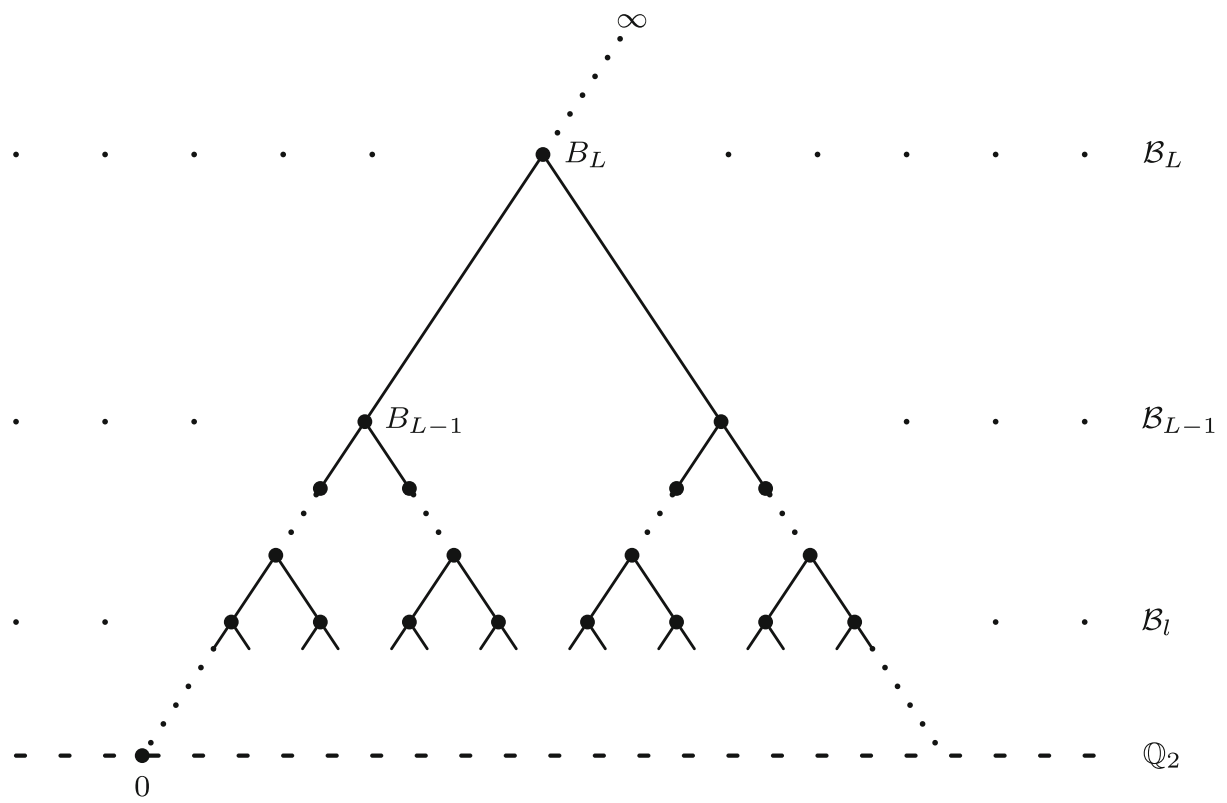

Fig. 1 Defining the arithmetic mean eigenvalue $\overline{\lambda_{l}}\left(B_{L}, \omega\right)$ 
and

$$
\frac{\bar{U}\left(B_{L}, \omega\right)-p}{\sqrt{2^{-L+l} p q}} \longrightarrow N(0,1) \quad \text { in law, }
$$

where $N(0,1)$ is the standard normal random variable.

For a given level $\mathcal{B}_{l}$ we define the arithmetic mean eigenvalue $\overline{\lambda_{l}}\left(B_{L}, \omega\right), L>l$, as

$$
\overline{\lambda_{l}}\left(B_{L}, \omega\right)=\frac{1}{2^{L-l}} \sum_{B: B \subset B_{L}, B \in \mathcal{B}_{l}} \lambda(B, \omega) .
$$

Applying Eqs. (4.7), (4.8) and the Eq. (4.4) we obtain the following result.

\section{Proposition 4.4 In the notation introduced above,}

$$
\overline{\lambda_{l}}\left(B_{L}, \omega\right) \longrightarrow 2^{-l}(1+\delta p) \quad P-a . s .,
$$

and

$$
\frac{\overline{\lambda_{l}}\left(B_{L}, \omega\right)-2^{-l}(1+\delta p)}{\delta \sqrt{2^{-L-l} p q}} \longrightarrow N(0,1) \text { in law }
$$

for any given level $\mathcal{B}_{l}$, and as $L \rightarrow \infty$.

Acknowledgments We are grateful to A. Grigor'yan, S. Molchanov, Ch. Pittet and W. Woess for their various comments and encouragement. We thank the participants of our seminar M. Krupski and W. Cygan for many fruitful discussions.

Open Access This article is distributed under the terms of the Creative Commons Attribution License which permits any use, distribution, and reproduction in any medium, provided the original author(s) and the source are credited.

\section{References}

1. Bendikov, A., Grigor'yan, A., Pittet, Ch.: On a class of Markov semigroups on discrete ultra-metric spaces. Potential Anal. 37, 125-169 (2012)

2. Bendikov, A., Grigor'yan, A., Pittet, Ch., Woess, W.: Isotropic Markov Semigroups On Ultrametric Spaces. arXiv: 1304.6271, to appear in Uspekhi Mat. Nauk (2014)

3. Bourbaki, N.: Elements of Mathematics. General Topology, Part II. Addison-Wesley, Hermann (1966)

4. Bovier, A.: The density of states in the Anderson model at weak disorder: A renormalization group analysis of the hierarchical model. J. Statist. Phys. 59(3-4), 745-779 (1990)

5. Dyson, F.J.: The dynamics of a disordered linear chain. Phys. Rev. 92, 1331-1338 (1953)

6. Dyson, F.J.: Existence of a phase-transition in a one-dimensional Ising ferromagnet. Comm. Math. Phys. 12, 91-107 (1969)

7. Engelking, R.: Theory of Dimensions, Finite and Infinite. Heldermann (1995)

8. Feldman, G.M.: Functional Equations and Characterization Problems on Locally Compact Abelian Groups. Tracts in Mathematics 5 European Mathematical Society (2008)

9. de Groot, J.: Non-Archimedean metrics in topology. Proc. Amer. Math. Soc. 7, 948-956 (1956)

10. Illanes, A., Nadler, S.B.Jr.: Hyperspaces: Fundamentals and Recent Advances. Marcell Dekker (1999)

11. Kritchevski, E.: Spectral localization in the hierarchical Anderson model. Proc. Amer. Math. Soc. 135, $1431-1440$ (2007)

12. Kritchevski, E.: Hierarchical Anderson model. Centre de Recherches Math. CRM Proc. and Lecture Notes, vol. 42 (2007)

13. Kritchevski, E.: Poisson Statistics of Eigenvalues in the Hierarchical Anderson Model. Ann. Henri Poincare 9, 685-709 (2008)

14. Lemin, A., Lemin, V.: On uniform rationalization of ultrametrics. Topology Proc. 22, 275-283 (1997)

15. Luukkainen, J., Movahedi-Lankarani, H.: Minimal bi-Lipschitz embedding dimension of ultrametric spaces. Fund. Math 144, 181-193 (1994) 
16. Molchanov, S.A.: Hierarchical random matrices and operators. Application to Anderson model. Proceedings of 6th Lukacs Symposium, pp. 179-194 (1996)

17. Vladimirov, V.S.: Generalized functions over the field of $p$-adic numbers. Uspekhi Mat. Nauk 43, 17-53, 239 (1988)

18. Vladimirov, V.S., Volovich, I.V.: p-adic Schrödinger-type equation. Lett. Math. Phys. 18, $43-53$ (1989)

19. Vladimirov, V.S., Volovich, I.V., Zelenov, E.I.: $p$-adic analysis and mathematical physics. Series on Soviet and East European Mathematics, vol.1, World Scientific Publishing Co., Inc., River Edge, NY (1994) 